\title{
Predicting substrates of the human breast cancer resistance protein using a support vector machine method
}

Eszter Hazai ${ }^{1}$, Istvan Hazai ${ }^{1}$, Isabelle Ragueneau-Majlessi ${ }^{2}$, Sophie P Chung ${ }^{2}$, Zsolt Bikadi ${ }^{1}$ and Qingcheng Mao ${ }^{2 *}$

\begin{abstract}
Background: Human breast cancer resistance protein (BCRP) is an ATP-binding cassette (ABC) efflux transporter that confers multidrug resistance in cancers and also plays an important role in the absorption, distribution and elimination of drugs. Prediction as to if drugs or new molecular entities are BCRP substrates should afford a costeffective means that can help evaluate the pharmacokinetic properties, efficacy, and safety of these drugs or drug candidates. At present, limited studies have been done to develop in silico prediction models for BCRP substrates. In this study, we developed support vector machine (SVM) models to predict wild-type BCRP substrates based on a total of 263 known BCRP substrates and non-substrates collected from literature. The final SVM model was integrated to a free web server.

Results: We showed that the final SVM model had an overall prediction accuracy of $\sim 73 \%$ for an independent external validation data set of 40 compounds. The prediction accuracy for wild-type BCRP substrates was 76\%, which is higher than that for non-substrates. The free web server (http://bcrp.althotas.com) allows the users to predict whether a query compound is a wild-type BCRP substrate and calculate its physicochemical properties such as molecular weight, logP value, and polarizability.
\end{abstract}

Conclusions: We have developed an SVM prediction model for wild-type BCRP substrates based on a relatively large number of known wild-type BCRP substrates and non-substrates. This model may prove valuable for screening substrates and non-substrates of BCRP, a clinically important ABC efflux drug transporter.

Keywords: Breast cancer resistance protein, Support vector machine, SVM, ATP-binding cassette, ABC transporter, in silico prediction, Substrate, BCRP, ABCG2

\section{Background}

Human breast cancer resistance protein (BCRP, gene symbol $A B C G 2)$ is an ATP-binding cassette (ABC) efflux drug transporter $[1,2]$. BCRP is one of the $\mathrm{ABC}$ transporters that confer resistance to a large number of structurally and chemically unrelated chemotherapeutic agents through ATP hydrolysis-dependent efflux transport of these drugs [2]. The substrates of BCRP have been rapidly expanding to include not only chemotherapeutics such as mitoxantrone, topotecan and imatinib, but also nonchemotherapeutic drugs such as prazosin, glyburide, nitrofurantoin and statins as well as non-therapeutic

\footnotetext{
*Correspondence: qmao@u.washington.edu

${ }^{2}$ Department of Pharmaceutics, School of Pharmacy, University of

Washington, Box 357610, Seattle, Washington 98195, USA

Full list of author information is available at the end of the article
}

compounds such as dietary flavonoids, porphyrins, estrone 3-sulfate, and the dietary carcinogen 2-amino-1-methyl-6phenylimidazo[4,5-b]pyridine [1,2]. BCRP is also highly expressed in organs important for the absorption (the small intestine), elimination (the liver and kidney), and distribution (the blood-brain and placental barriers) of drugs and xenobiotics [3], and has recently been recognized by the FDA as one of the most important drug transporters involved in clinically relevant drug disposition and drug-drug interactions [4]. Due to the clinical importance of BCRP in drug resistance and drug disposition, it should be of high value to develop cost-effective methods for evaluation of transport of drugs or drug candidates by BCRP so that the pharmacokinetics, efficacy, safety, and tissue levels of these compounds may be

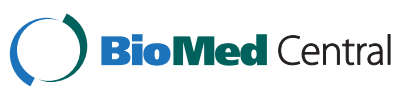


predicted. One of such methods would be the development of in silico models for prediction of BCRP substrates.

Indeed, in the recent years, in silico prediction models have emerged into the pipeline of drug discovery which allow initial screening and selection of promising compounds from chemical libraries and large databases. In addition, these models could provide information concerning the mechanism of protein-ligand interactions. In silico methods for prediction of protein-ligand interactions including transport characteristics can be divided into ligand-based and protein structure-based approaches. With protein structure-based methods such as molecular docking, structures and physicochemical characteristics of an intermolecular complex formed between interacting protein and ligand could be predicted if high resolution structures of both the protein and the ligand under question are available. High resolution structures of BCRP have not been resolved. Homology models of BCRP have recently been developed and await further experimental validation [1,5]. Although these homology models can be used for docking calculations and interpretation of biochemical data, results obtained are unlikely reliable for drug design and screening. In contrast, ligand-based methods based on structural similarity of ligands to known substrates generally yield much greater prediction accuracies than protein structure-based methods.

Among ligand-based methods, one common approach is to develop quantitative structure-activity relationship models (SAR and QSAR). The objective of SAR and QSAR analysis is to establish a correlation between descriptors which represent information of molecular structures of ligands and biological activities for a series of biologically and structurally characterized compounds. Various SAR and QSAR models for BCRP inhibitors have been published [6-8]. Several SAR and QSAR studies suggest that lipophilicity of ligands is a good predictor for BCRP inhibition [9-11], but other studies argue that this property is not significant $[12,13]$. A planar structure of inhibitors seems to be necessary for binding to the active site of BCRP $[9,14,15]$. With respect to prediction of BCRP substrates, only one SAR study of camptothecin analogues revealed that hydrogen bond formation might be important for substrate recognition by BCRP [16]. One common feature of these SAR and QSAR models is that these models are usually built using a congeneric series of molecules and thus may not be valid for other classes of compounds. For this reason, more sophisticated techniques are required for classification of BCRP ligands.

Another ligand-based approach is to use statistical learning methods to predict features based on properties of examples, and compounds of any chemical structures can be used. Of these methods, the support vector machine (SVM) method is most frequently used and has proved valuable in a wide range of applications. SVM has gained popularity in the chemo- and bioinformatics field due to its ability to classify objects into two classes based on their structural features. In particular, the SVM method was useful for classification of molecules as substrates or non-substrates of enzymes or transporters. For example, several studies have been reported for prediction of substrates and non-substrates of P-glycoprotein (P-gp) using SVM with generally greater than $70 \%$ prediction accuracies [17-20]. Zhong et al. recently reported a genetic algorithm-conjugate gradient-support vector machine (GA-CG-SVM) procedure for prediction of BCRP substrates and non-substrates [21]. Although these studies are highly valuable, the scientific community has no open access to most of these published in silico models. There are a few SVM-based free web servers for predicting substrates and non-substrates of certain enzymes and transporters. For example, Mishra et al. reported a web server for cytochrome $\mathrm{P} 450$ enzymes [22], and our laboratories published a free web server for prediction of $\mathrm{P}$-gp substrates and nonsubstrates using the SVM method (http://pgp.althotas. com) [20].

Therefore, in the present study, we have compiled a relatively large data set of BCRP substrates and nonsubstrates collected from literature and developed an SVM-based in silico model for prediction of wild-type BCRP substrates and non-substrates. This prediction model has been integrated into a free web server (http:// bcrp.althotas.com) which allows the users to predict the capability of wild-type BCRP to transport the query ligands and calculate their physiochemical properties including molecular weight, $\log \mathrm{P}$ value, and polarizability.

\section{Methods \\ Data set}

All known wild-type BCRP substrates and nonsubstrates used in this study were taken from published data in the literature. Information for some of these compounds in the data set was obtained through searching the University of Washington Metabolism \& Transport Drug Interaction Database (http://www. druginteractioninfo.org/). This data set is based on results of in vitro transport assays such as the membrane vesicle uptake assay, the efflux assay using intact mammalian cells over-expressing BCRP, and transwell transport assay using MDCKII/BCRP cells. Results from in vitro drug resistance assays were also used. However, results from drug-stimulated ATPase assays were not used because many substrates do not stimulate ATPase activity of BCRP. In the case of conflicting evidence, only the results confirmed by at least two independent 
studies were accepted. This data set contains 164 BCRP substrates and 99 non-substrates with highly diverse chemical structures. We noticed that 60 out of the 164 substrates had multiple reports. However, only about 9 out of the 99 non-substrates had multiple reports. It is worth noting that the drug-selected BCRP mutants with amino acid substitutions at position 482 exhibit altered substrate specificity. For example, doxorubicin, rhodamine 123 and LysoTracker Green are substrates of the mutant R482G or R482T, but cannot be efficiently transported by wild-type BCRP [23-25]. Therefore, such compounds were classified as non-substrates of wildtype BCRP which was the subject of this study. Of the 263 compounds (164 substrates and 99 non-substrates), 223 compounds (139 substrates and 84 non-substrates) were randomly used in the training and test subsets in various training/test ratios, and 40 compounds (25 substrates and 15 non-substrates) were defined as the independent external validation subset. All compounds are listed in Additional file 1: Table S1. The chemical structures of all these molecules are shown in two sdf files provided as Additional files 2 and 3 which can be viewed using the free MarvinView software (http://www. chemaxon.com/products/marvin/marvinview/).

\section{Support vector machine (SVM)}

The SVM method we used in this study is essentially the same as previously described [20]. Briefly, the standard procedure of classification by SVM can be divided into four stages. In the first stage, all compounds in the data set were defined as substrates and non-substrates of wild-type BCRP. Then, the molecules were characterized using molecular descriptors. The data set was then split into the training and test subsets, and an independent external validation subset was also created. In the second stage, the compounds in the training set were presented as points in a high-dimensional space according to their molecular descriptors. In this high-dimensional space, a hyperplane was determined to separate objects into substrate and non-substrate groups. Since various hyperplanes allow separation of objects, a hyperplane that maximizes the margin needs to be constructed. In the third stage, the models constructed using the training data set were used to calculate prediction accuracy for a test set to evaluate the models. Finally, the models were validated using the independent external data set.

Chemical structures of all wild-type BCRP substrates or non-substrates used in this study were downloaded from the PubChem Database (http://pubchem.ncbi.nlm. nih.gov). Some compounds were extracted from the original publications and redrawn by means of MarvinView (ChemAxon, Budapest, Hungary). All molecules were subject to geometry optimization using the Molconvert software (ChemAxon, Budapest, Hungary), which applies the Dreiding molecular mechanics force field, and to calculation of the Gasteiger partial charges [26]. The DragonX software (www.talete.mi.it) was used to calculate a total of 3250 molecular descriptors for each molecule. The descriptors with more than $80 \%$ zero values and too small standard deviation values (less than 3\%) were eliminated. The Libsvm software (www.csie.ntu. edu.tw/ cjlin/libsvm/) was then used for SVM calculations. Linear, polynomial, and radial basis function (RBF) kernels were tested in this study. RBF is calculated using the equation $K(x i, x j)=\exp \left(-\gamma\|x i-x j\|^{2}\right)$, where $\gamma$ is a kernel parameter, $x i$ and $x j$ are instance label pairs, and $\mathrm{K}$ is the kernel function. The prediction power of SVM is greatly influenced by the selection of kernel, the kernel parameter $\gamma$, and soft margin parameter $\mathrm{C}$.

The best combination of $\mathrm{C}$ and $\gamma$ was selected by a grid-search with exponentially growing sequences of $\mathrm{C}$ and $\gamma$. Each combination of parameter choices was checked, and the parameters with the best validation accuracy were selected. After the best parameters $C$ and $\gamma$ were found, the whole training set was trained again to generate the final model. The feature selection tool fselect.py (http://www.csie.ntu.edu.tw/ cjlin/libsvmtools) provided by the Libsvm developer was used to measure the relative importance of each feature. For each feature, an F-score can be calculated using fselect.py. Generally, the larger the F-score, the more likely the feature is discriminative. Therefore, F-score was used as a feature selection criterion. Features with high F-scores were selected and then SVM was applied. High F-score features were gradually added until the validation accuracy decreased. Descriptors were checked for their correlation. Among the descriptors with a correlation of 0.9 , the descriptors with higher F-scores were kept for further SVM calculations. Prediction power of the abovedescribed SVM method was evaluated based on the number of true positive (TP), true negative (TN), false positive (FP), and false negative (FN) predictions. Additional parameters that are widely used, namely accuracy (ACC), sensitivity (SE), specificity (SP), and the Matthews correlation coefficient (MCC), were also calculated using the following equations:

$$
\begin{array}{r}
\mathrm{ACC}=[(\mathrm{TP}+\mathrm{TN}) /(\mathrm{TP}+\mathrm{TN}+\mathrm{FP}+\mathrm{FN})] \times 100 \\
\mathrm{SE}=[\mathrm{TP} /(\mathrm{TP}+\mathrm{FN})] \times 100 \\
\mathrm{SP}=[\mathrm{TN} /(\mathrm{FP}+\mathrm{TN})] \times 100 \\
\mathrm{MCC}=(\mathrm{TP} \times \mathrm{TN}-\mathrm{FP} \times \mathrm{FN}) /[(\mathrm{TP}+\mathrm{FN})(\mathrm{TP}+\mathrm{FP}) \\
(\mathrm{TN}+\mathrm{FP})(\mathrm{TN}+\mathrm{FN})]^{1 / 2}
\end{array}
$$

\section{The web server}

The best prediction model generated using the SVM method described above has been integrated into a free web server (http://bcrp.althotas.com). This web server allows the users to predict as to whether a query 
compound is likely to be a BCRP substrate. The chemical structure of the query compounds can be uploaded or drawn in by the users using the built-in Chemaxon Marvin Java applet. The web server is linked to PubChem so that any query compounds can be directly retrieved with text search. Any compounds of interest can be searched by their names, uploaded in PDB, mol, mol2, hin, or SMILES format or drawn in using a Marvin applet by the users. Structural conversions and 3-dimensional geometry optimization by the Dreiding method are carried out using the Molconvert software. Two-dimensional and 3-dimensional molecular descriptors are calculated using the DragonX software.

\section{Results and discussion}

Since SVM tends to find a linear separating hyperplane with the maximal margin in a high-dimensional space by using a penalty parameter of the error term and a kernel function, we first investigated the influence of kernel function on the performance parameters. SVM prediction performance parameters of 100 runs with different kernel functions (linear, polynomial, and RBF) are provided in Table 1 . The data shown in Table 1 were obtained using a training set of 167 compounds, a test set of 56 compounds, and an external validation set of 40 compounds. The test set was used for choosing the best kernel function, and test performances were used as the criteria for selecting the best kernel. It should be emphasized that the external validation set was not used in the model building steps. It appeared that polynomial kernel function produced generally lower prediction accuracy compared to linear kernel function and RBF. Although performance parameters associated with linear

Table 1 The mean values of SVM prediction performance parameters of 100 runs using various kernels

\begin{tabular}{llllll}
\hline Kernel & Category & ACC & SE & SP & MCC \\
\hline Linear & Training & 80.7 & 90.4 & 64.8 & 0.581 \\
& Test & 68.8 & 82.1 & 46.6 & 0.312 \\
& External & 70.7 & 77.6 & 59.1 & 0.375 \\
Polynomial & Training & 79.2 & 96.4 & 50.7 & 0.506 \\
& Test & 65.8 & 86.8 & 30.8 & 0.198 \\
& External & 66.1 & 81.9 & 39.8 & 0.174 \\
RBF & Training & 84.5 & 93.8 & 69.1 & 0.665 \\
& Test & 69.7 & 83.3 & 47.2 & 0.332 \\
& External & 70.9 & 77.7 & 59.6 & 0.382 \\
\hline
\end{tabular}

ACC, accuracy (overall prediction accuracy); SP, specificity (prediction accuracy for the non-substrates); $\mathrm{SE}$, sensitivity (prediction accuracy for the substrates); MCC, the Matthews correlation coefficient (a more balanced prediction parameter than ACC). The external data set was only used to validate the prediction power of the models constructed, and was not used for model selection. and RBF kernels were comparable, RBF provided slightly better prediction results. This is consistent with a general practice that RBF is the most popular choice of kernel function in SVM. Based on results of this preliminary evaluation, only RBF was used in further calculations.

Due to the limited number of currently known wildtype BCRP substrates and non-substrates, if more compounds are used in the training set, fewer compounds can be used in the test set, likely resulting in less reliable test prediction outcome. Therefore, we next investigated the influence of the number of compounds in the training and test sets on prediction accuracy. The results of SVM calculations performed with varying training/test set ratios are shown in Table 2. Overall, we did not observe significant differences in the performance parameters with different training/test ratios. However, the MCC values at the training/test ratios of $0.75 / 0.25 ; 0.70 /$ 0.30 and $0.60 / 0.40$ appeared to be comparable and slightly better than those at other ratios. Similar to the kernel selection, only the test and training data sets were

Table 2 Performance parameters of 100 runs using various ratios of training/test sets

\begin{tabular}{|c|c|c|c|c|c|}
\hline Training/Test ratio & Category & $\mathrm{ACC}$ & SE & $\mathrm{SP}$ & $\mathrm{MCC}$ \\
\hline \multirow[t]{3}{*}{$0.5 / 0.5$} & Training & 83.8 & 94.6 & 65.8 & 0.649 \\
\hline & Test & 67.8 & 82.2 & 44.0 & 0.288 \\
\hline & External & 69.5 & 78.3 & 54.7 & 0.344 \\
\hline \multirow[t]{3}{*}{$0.6 / 0.4$} & Training & 85.1 & 94.7 & 69.1 & 0.678 \\
\hline & Test & 69.0 & 83.2 & 45.5 & 0.315 \\
\hline & External & 70.8 & 79.0 & 57.1 & 0.372 \\
\hline \multirow[t]{3}{*}{$0.7 / 0.3$} & Training & 83.4 & 93.3 & 67.1 & 0.642 \\
\hline & Test & 71.1 & 84.2 & 49.1 & 0.360 \\
\hline & External & 70.8 & 78.4 & 58.1 & 0.375 \\
\hline \multirow[t]{3}{*}{$0.75 / 0.25$} & Training & 84.5 & 93.8 & 69.1 & 0.665 \\
\hline & Test & 69.7 & 83.3 & 47.2 & 0.332 \\
\hline & External & 70.9 & 77.7 & 59.6 & 0.382 \\
\hline \multirow[t]{3}{*}{$0.8 / 0.2$} & Training & 83.6 & 93.5 & 67.1 & 0.644 \\
\hline & Test & 70.4 & 83.6 & 48.7 & 0.35 \\
\hline & External & 70.6 & 77.5 & 59.1 & 0.376 \\
\hline \multirow[t]{3}{*}{$0.85 / 0.15$} & Training & 82.9 & 93.5 & 65.1 & 0.627 \\
\hline & Test & 70.3 & 85.1 & 46.4 & 0.347 \\
\hline & External & 70.9 & 78.5 & 58.1 & 0.376 \\
\hline
\end{tabular}

The total number of molecules used in the training and test data sets were 223. The number of molecules in the external validation data set was 40 . ACC, accuracy (overall prediction accuracy); SP, specificity (prediction accuracy for the non-substrates); $\mathrm{SE}$, sensitivity (prediction accuracy for the substrates); MCC, the Matthews correlation coefficient (a more balanced prediction parameter than ACC). The external data set was only used to validate the prediction power of the models constructed, and was not used for model selection. 
used for model construction, and the external validation data set was only used for validation of the models constructed. Thus, the training/test ratio of $0.75 / 0.25$ was chosen for further calculations in order to maximize the chemical space occupied by the molecules in the training set.

There is no general rule regarding selection of the best SVM prediction model. The run that provides the highest prediction accuracy for the training set may be selected. However, such an approach could be misleading because a model with the highest prediction accuracy (or Matthews correlation coefficient) for a training set does not necessarily produce the highest prediction accuracy (or Matthews correlation coefficient) neither for the test set nor for the independent external validation set due to a phenomenon called overfitting. It is therefore necessary to consider prediction characteristics of both the training and test sets when the best SVM model is to be selected. In our study for prediction of Pgp substrates [20], we proposed the following approach. First, the differences in prediction accuracy between the training and test sets were calculated, and the models with the smallest difference were taken into account. Second, of the models with the smallest difference in prediction accuracy between the training and test sets, those built with the smallest number of molecular descriptors were considered because inclusion of too many descriptors may again produce overfitted models and the inclusion of unnecessary or irrelevant descriptors creates noise in the model. We showed that classification of individual compounds in the independent external validation set as substrates or non-substrates of P-gp was very similar among the potentially best models [20]. Using the same approach, the best SVM prediction model was selected for substrates of wild-type BCRP and the prediction performance parameters of the selected model are shown in Table 3 . The selected model showed an overall prediction accuracy of $\sim 73 \%$ for the external validation data set. Also, wild-type BCRP substrates were generally predicted with a higher accuracy than non-substrates (Table 3). In the SVM prediction,

Table 3 Prediction power of the selected SVM model

\begin{tabular}{lcccccccc}
\hline & TP & FN & TN & FP & ACC & SE & SP & MCC \\
\hline Training set & 89 & 15 & 38 & 25 & 76.0 & 85.6 & 60.3 & 0.478 \\
Test set & 31 & 4 & 11 & 10 & 75.0 & 88.6 & 52.4 & 0.448 \\
External set & 19 & 6 & 10 & 5 & 72.5 & 76.0 & 66.7 & 0.422 \\
\hline
\end{tabular}

$\mathrm{TP}$, true positive; $\mathrm{TN}$, true negative; $\mathrm{FP}$, false positive; $\mathrm{FN}$, false negative; $\mathrm{ACC}$, accuracy (overall prediction accuracy); SP, specificity (prediction accuracy for the non-substrates); $\mathrm{SE}$, sensitivity (prediction accuracy for the substrates); MCC, the Matthews correlation coefficient (a more balanced prediction parameter than ACC). there is generally a borderline region where the structural properties of substrates and non-substrates are very similar, and therefore compounds in this borderline region cannot be separated by their structural properties alone. In our model, molecules in this borderline region were mainly predicted as substrates. This may contribute to the higher prediction accuracy for substrates.

Classification of individual compounds as wild-type BCRP substrates or non-substrates was very similar among the 10 selected models in the first step. Table 4 shows the overlap in classification (i.e. the percentage of compounds that were identically predicted by the compared models) among these 10 models. As can be seen in Table 4, classification of compounds to be wild-type BCRP substrates or non-substrates in different models were highly similar, that is, the overlap values varied between $81.4 \%$ and $94.3 \%$, with an average of $88.5 \%$. For example, $85.93 \%$ of compounds were predicted to be in the same category (substrates or non-substrates) by the model 87 compared to the model 63 , which has the highest prediction accuracy. This finding suggests that, to obtain reliable predictions, selection of the best model is likely not as important as, for example, the compilation of high quality data sets. In this regard, we would like to point out that the data set collected in this study regarding whether a specific compound is a substrate of wild-type BCRP could be obtained using different transport methods. This does not affect the prediction accuracy of our model as long as the different transport methods produce the same result as to if the compound is a BCRP substrate. This is because the SVM model only makes qualitative (substrates or non-substrates), not quantitative (e.g., transport capacity) prediction.

Recently, an SVM study based on a different data set was published by Zhong et al. [21] and reported a higher overall prediction accuracy for BCRP substrates and nonsubstrates ( $85 \%$ for the test set). It should be noted that the compounds used by Zhong et al. were only divided into two sets, namely a training set and a test set, without an independent external validation data set. Also, the test set used by Zhong et al. was not independent when it was used for the selection of the best model. Therefore, their results cannot be directly compared to the data of this study. This is because, besides the training and test sets, we also used an independent external validation data set to evaluate prediction outcome and calculate prediction accuracies of the selected best model. Moreover, certain compounds in the data sets of Zhong et al. were actually the same under different names (e.g., folic acid versus vitamin B9 and daunomycin versus daunorubicin). Additionally, a number of compounds were classified as BCRP substrates (e.g., daunorubicin, rhodamine 123, LysoTracker Green, and epirubicin) by Zhong et al., but as non-substrates in this study as explained in the Methods section. 
Table 4 Overlap of classification in 10 experimental models

\begin{tabular}{|c|c|c|c|c|c|c|c|c|c|c|}
\hline Experimental model & 98 & 87 & 77 & 63 & 91 & 45 & 42 & 62 & 7 & 73 \\
\hline 98 & 100 & 86.31 & 87.07 & 86.69 & 84.41 & 81.37 & 87.07 & 87.07 & 87.45 & 86.69 \\
\hline 87 & & 100 & 87.83 & 85.93 & 87.45 & 81.37 & 87.83 & 87.07 & 87.45 & 90.49 \\
\hline 77 & & & 100 & 89.73 & 94.30 & 87.45 & 91.64 & 92.34 & 89.73 & 91.26 \\
\hline 63 & & & & 100 & 87.83 & 84.79 & 88.21 & 89.73 & 89.35 & 88.59 \\
\hline 91 & & & & & 100 & 88.59 & 88.97 & 94.30 & 91.64 & 92.40 \\
\hline 45 & & & & & & 100 & 85.93 & 88.21 & 84.79 & 84.79 \\
\hline 42 & & & & & & & 100 & 91.64 & 92.78 & 92.02 \\
\hline 62 & & & & & & & & 100 & 91.26 & 91.26 \\
\hline 7 & & & & & & & & & 100 & 91.26 \\
\hline 73 & & & & & & & & & & 100 \\
\hline
\end{tabular}

The overall prediction accuracies (ACC) of the 10 experimental models $98,87,77,63,91,45,42,62,7$, and 73 were $78.33 \%, 75.29 \%, 76.81 \%, 80.23 \%, 75.67 \%$,

$70.34 \%, 76.05 \%, 75.29 \%, 78.71 \%$, and $77.95 \%$, respectively.

We found that the final SVM model selected in this study used the molecular descriptors shown in Table 5. That is, the following descriptors were found to be used in the final model: mean information index on atomic composition (AAC), spherosity (SPH), Morse signals, and a mass weighed Gateway descriptor. These descriptors suggest that the 3-dimensional structure of a substrate is likely the determining factor for BCRP/substrate interactions. The results of classification by this SVM model for all compounds used in this study are shown in Additional file 1: Table S1.

In order to make the SVM model publicly available, we developed a free web server (http://bcrp.althotas. com). This web server enables the users to predict if a query compound is a BCRP substrate based on the selected SVM prediction model of this study.

\section{Conclusions}

In summary, $\mathrm{BCRP}$ is an $\mathrm{ABC}$ drug transporter that confers multidrug resistance in cancers and plays an important role in drug disposition. Therefore, it is important to develop in silico prediction models for BCRP substrates that could be used as cost-effective tools for screening of

Table 5 List of molecular descriptors found to be used by the selected SVM model

\begin{tabular}{ll}
\hline $\begin{array}{l}\text { Dragon } \\
\text { abbreviation }\end{array}$ & Molecular descriptor \\
\hline AAC & Mean information index on atomic composition \\
SPH & spherosity \\
Mor17m & 3D Morse signal 17/weighed by mass \\
Mor25m & 3D Morse signal 25/weighed by mass \\
R2m & Gateway R autocorrelation of lag2 weighed by \\
& mass \\
\hline
\end{tabular}

drug candidates in early drug discovery stage and for identification of BCRP substrates among existing drugs so that potential drug-drug interactions may be predicted. In the present study, using a carefully defined and relatively large data set with 263 known wild-type BCRP substrates and non-substrates, we have developed an SVM model for prediction of wild-type BCRP substrates and non-substrates with an overall prediction accuracy of $\sim 73 \%$ for an independent external validation data set of 40 compounds. The prediction accuracy for wild-type BCRP substrates was $\sim 76 \%$, which is higher than that for non-substrates. The molecular descriptors used by this SVM model suggest that the 3-dimensional structure of a compound is possibly a predominant factor in determining $\mathrm{BCRP} /$ substrate interactions. This SVM prediction model has been integrated into a web server (http://bcrp.althotas.com) which is freely available to the scientific community. We believe that availability of such a prediction model will facilitate drug discovery as well as basic research investigating the role of BCRP in drug transport.

\section{Additional files}

Additional file 1: All the wild-type BCRP substrates and nonsubstrates used in this study and classification of these compounds by the selected SVM prediction model developed in this study are shown in the supplemental Table S1.

Additional file 2: The chemical structures of all wild-type BCRP substrates are shown in this sdf file which can be viewed using the free MarvinView software (http://www.chemaxon.com/products/ marvin/marvinview/). The order of molecules in the sdf file is according to the supplementary Table S1.

Additional file 3: The chemical structures of all wild-type BCRP non-substrates are shown in this sdf file which can be viewed using the free MarvinView software (http://www.chemaxon.com/products/ marvin/marvinview/). The order of molecules in the sdf file is according to the supplementary Table S1. 


\section{Abbreviations}

ABC: ATP-binding cassette; BCRP: Breast cancer resistance protein; ABCG2: The second member of ABC transporter subfamily G; SVM: Support vector machine; ACC: Accuracy (overall prediction accuracy); SP: Specificity (prediction accuracy for non-substrates); SE: Sensitivity (prediction accuracy for substrates); MCC: Matthews correlation coefficient (a more balanced prediction parameter than ACC); SD: Standard deviation; TP: True positive; FP: False positive; TN: True negative; FN: False negative.

\section{Competing interests}

The authors declare that they have no competing interests.

\section{Authors' contributions}

EH: data analysis and interpretation, manuscript preparation, and final approval of the version to be published; $\mathrm{IH}$ : collection of data sets, data analysis and interpretation, manuscript preparation; SC: collection of data sets and critical revision of the manuscript; IR-M: collection of data sets and critical revision of the manuscript; ZB: data analysis and interpretation, manuscript preparation, and final approval of the version to be published; QM: collection of data sets, data analysis and interpretation, manuscript preparation, and final approval of the version to be published. All authors read and approved the final manuscript.

\section{Acknowledgements}

This work was supported by the Hungarian State and the European Union (European Regional Development Fund), under the aegis of New Hungary Development Plan (KMOP-1.1.1-09/1-2009-0044), and by a grant from the National Institutes of Health, GM073715 (to QM).

\section{Author details}

${ }^{1}$ Virtua Drug Ltd., Csalogany Street 4, Budapest H-1015, Hungary. ${ }^{2}$ Department of Pharmaceutics, School of Pharmacy, University of Washington, Box 357610, Seattle, Washington 98195, USA.

Received: 6 November 2012 Accepted: 12 April 2013 Published: 15 April 2013

\section{References}

1. Ni Z, Bikadi Z, Rosenberg MF, Mao Q: Structure and function of the human breast cancer resistance protein (BCRP/ABCG2). Curr Drug Metab 2010, 11(7):603-617.

2. Natarajan $K$, Xie $Y$, Baer MR, Ross DD: Role of breast cancer resistance protein (BCRP/ABCG2) in cancer drug resistance. Biochem Pharmacol 2012, 83(8):1084-1103.

3. Maliepaard M, Scheffer GL, Faneyte IF, van Gastelen MA, Pijnenborg AC, Schinkel AH, van De Vijver MJ, Scheper RJ, Schellens JH: Subcellular localization and distribution of the breast cancer resistance protein transporter in normal human tissues. Cancer Res 2001, 61(8):3458-3464.

4. Giacomini KM, Huang SM, Tweedie DJ, Benet LZ, Brouwer KL, Chu X, Dahlin A, Evers R, Fischer V, Hillgren KM, et al: Membrane transporters in drug development. Nat Rev Drug Discov 2010, 9(3):215-236.

5. Rosenberg MF, Bikadi Z, Chan J, Liu X, Ni Z, Cai X, Ford RC, Mao Q: The human breast cancer resistance protein (BCRP/ABCG2) shows conformational changes with mitoxantrone. Structure 2010, 18(4):482-493.

6. Gandhi YA, Morris ME: Structure-activity relationships and quantitative structure-activity relationships for breast cancer resistance protein (ABCG2). AAPS J 2009, 11(3):541-552.

7. Ishikawa T, Hirano H, Saito H, Sano K, Ikegami Y, Yamaotsu N, Hirono S: Quantitative structure-activity relationship (QSAR) analysis to predict drug-drug interactions of $A B C$ transporter $A B C G 2$. Mini Rev Med Chem 2012, 12(6):505-514

8. Nicolle E, Boumendjel A, Macalou S, Genoux E, Ahmed-Belkacem A, Carrupt PA, Di Pietro A: QSAR analysis and molecular modeling of ABCG2-specific inhibitors. Adv Drug Deliv Rev 2009, 61(1):34-46.

9. Zhang S, Yang X, Coburn RA, Morris ME: Structure activity relationships and quantitative structure activity relationships for the flavonoidmediated inhibition of breast cancer resistance protein. Biochem Pharmacol 2005, 70(4):627-639.

10. van Loevezijn A, Allen JD, Schinkel AH, Koomen GJ: Inhibition of BCRPmediated drug efflux by fumitremorgin-type indolyl diketopiperazines. Bioorg Med Chem Lett 2001, 11(1):29-32.
11. Matsson P, Englund G, Ahlin G, Bergstrom CA, Norinder U, Artursson P: A global drug inhibition pattern for the human ATP-binding cassette transporter breast cancer resistance protein (ABCG2). J Pharmacol Exp Ther 2007, 323(1):19-30.

12. Cramer J, Kopp S, Bates SE, Chiba P, Ecker GF: Multispecificity of drug transporters: probing inhibitor selectivity for the human drug efflux transporters ABCB1 and ABCG2. ChemMedChem 2007, 2(12):1783-1788.

13. Pick $A$, Muller $H$, Wiese $M$ : Structure-activity relationships of new inhibitors of breast cancer resistance protein (ABCG2). Bioorg Med Chem 2008, 16(17):8224-8236.

14. Ahmed-Belkacem A, Pozza A, Munoz-Martinez F, Bates SE, Castanys S, Gamarro F, Di Pietro A, Perez-Victoria JM: Flavonoid structure-activity studies identify 6-prenylchrysin and tectochrysin as potent and specific inhibitors of breast cancer resistance protein ABCG2. Cancer Res 2005, 65(11):4852-4860.

15. Ahmed-Belkacem A, Macalou S, Borrelli F, Capasso R, Fattorusso E, Taglialatela-Scafati O, Di Pietro A: Nonprenylated rotenoids, a new class of potent breast cancer resistance protein inhibitors. J Med Chem 2007, 50(8):1933-1938.

16. Nakagawa H, Saito H, Ikegami Y, Aida-Hyugaji S, Sawada S, Ishikawa T: Molecular modeling of new camptothecin analogues to circumvent ABCG2-mediated drug resistance in cancer. Cancer Lett 2006, 234(1):81-89.

17. Wang Z, Chen $Y$, Liang $H$, Bender A, Glen RC, Yan A: P-glycoprotein substrate models using support vector machines based on a comprehensive data set. J Chem Inf Model 2011, 51(6):1447-1456.

18. Xue Y, Yap CW, Sun LZ, Cao ZW, Wang JF, Chen YZ: Prediction of P-glycoprotein substrates by a support vector machine approach. J Chem Inf Comput Sci 2004, 44(4):1497-1505.

19. Huang J, Ma G, Muhammad I, Cheng Y: Identifying P-glycoprotein substrates using a support vector machine optimized by a particle swarm. J Chem Inf Model 2007, 47(4):1638-1647.

20. Bikadi Z, Hazai I, Malik D, Jemnitz K, Veres Z, Hari P, Ni Z, Loo TW, Clarke DM, Hazai E, et al: Predicting P-glycoprotein-mediated drug transport based on support vector machine and three-dimensional crystal structure of P-glycoprotein. PLOS One 2011, 6(10):e25815.

21. Zhong L, Ma CY, Zhang $H$, Yang LJ, Wan HL, Xie QQ, Li LL, Yang SY: A prediction model of substrates and non-substrates of breast cancer resistance protein (BCRP) developed by GA-CG-SVM method. Comput Biol Med 2011, 41(11):1006-1013.

22. Mishra NK, Agarwal S, Raghava GP: Prediction of cytochrome P450 isoform responsible for metabolizing a drug molecule. BMC Pharmacol 2010, 10:8.

23. Honjo Y, Hrycyna CA, Yan QW, Medina-Perez WY, Robey RW, van de Laar A, Litman T, Dean $M$, Bates SE: Acquired mutations in the MXR/BCRP/ABCP gene alter substrate specificity in MXR/BCRP/ABCP-overexpressing cells. Cancer Res 2001, 61(18):6635-6639.

24. Robey RW, Honjo Y, Morisaki K, Nadjem TA, Runge S, Risbood M, Poruchynsky MS, Bates SE: Mutations at amino-acid 482 in the ABCG2 gene affect substrate and antagonist specificity. Br J Cancer 2003, 89(10):1971-1978.

25. Ozvegy-Laczka C, Koblos G, Sarkadi B, Varadi A: Single amino acid (482) variants of the $A B C G 2$ multidrug transporter: major differences in transport capacity and substrate recognition. Biochim Biophys Acta 2005, 1668(1):53-63

26. Gasteiger JMM: Iterative partial equalization of orbital electronegativity a rapid access to atomic charges. Tetrahedron 1980, 36:3219-3228.

doi:10.1186/1471-2105-14-130

Cite this article as: Hazai et al:: Predicting substrates of the human breast cancer resistance protein using a support vector machine method. BMC Bioinformatics 2013 14:130. 